\title{
Activation of Retrotransposon in Red Alga by Underwater Pulsed Discharge
}

\author{
K. Hirayama, D. Wang*, M. Matsuda, X. Lin, T. Namihira, H. Takano, S. Takio \\ AND H. AKIYAMA \\ Priority Organization for Innovation and Excellence, Kumamoto University \\ 2-39-1 Kurokami 860-8555, Kumamoto, Japan
}

\begin{abstract}
Retrotransposons are mobile genetic elements that accomplish transposition via an RNA intermediate. These elements can be transcriptionally activated by stress factors, such as UV light, ozone, pathogens, wounding and drought. A red alga, Porphyra yezoensis has recently been recognized as a model plant for fundamental and applied study in marine biological science. In this paper, pulsed streamer-like discharge in liquid was used as a new stress condition, and the transcriptional levels of retrotransposon and functional genes of a red alga, Porphyra yezoensis was investigated. From the results of reverse transcription-polymerase chain reaction (RT-PCR) experiments, it is clear that the transcriptions of retrotransposon and functional genes were activated by applying pulsed streamer-like discharge.
\end{abstract}

PACS numbers: 52.80.Wq

\section{Introduction}

Retrotransposons are mobile genetic elements that accomplish transposition via an RNA intermediate that is reverse transcribed before integration into a new location within the host genome [1-4]. They are ubiquitous in eukaryotes organisms and constitute a major portion of the nuclear genome (often more than half of the total DNA) in plants. Their biological impact on the evolution through creation of genomic variability and speciation of various eukaryotes was speculated. Functional genes are genetic elements that can generate proteins. In this paper, we introduce three functional genes, bromoproxidase, superoxide dismutase and protease gene family. Bromoproxidase is enzyme that catalyses the bromination of organic matrix. Superoxide dismutase is enzyme that catalyses conversion of super oxide to hydrogen peroxide and oxygen. Protease gene family are enzymes that hydrolyse peptides and proteins. Streamer-like discharges in liquid are known to generate extremely intense electric fields at the tip of streamers, as well as high energy electrons, ozone, other chemically active species, ultraviolet rays and shock waves [5]. In the work, the effect of the multiple stresses by underwater streamer-like discharge on transcriptional activations of the retorotransposon and functional genes of red alga was investigated.

\section{Experimental method}

\subsection{Pulsed discharge application system}

Figure 1 shows the schematic diagram of the pulsed power generator used in this work. The generator con-

\footnotetext{
* corresponding author; e-mail: douyan@kumamoto-u.ac.jp
}

sisted of a dc source (E, HDV-50K3US, Pulse Electronic Engineering Co., Japan), the Blumlein type pulse forming network (B-PFN) and a pulse transformer (PT). B-PFN had 12 stages of $L C$ ladder, which were composed of $2.7 \mathrm{nF}$ capacitor and $0.1 \mu \mathrm{H}$ inductor. The characteristic impedance $(2 \sqrt{L / C})$ and the pulse width $(2 N \sqrt{L C})$ of B-PFN, calculated from capacitance $(C)$ and inductance $(L)$ of $L C$ ladder, and number $(N)$ of $L C$ ladder stages, were approximately $12.2 \Omega$ and $0.4 \mu \mathrm{s}$, respectively. PT had 6 of the winding ratio (primary windings: secondary windings $=1: 6)$. Therefore, the total characteristic impedance of the generator was computed at $440 \Omega$. Figure 2 shows the configuration of the discharge chamber used in this experiment. The needle to cylinder electrode was utilized as the discharge chamber. The needle electrode with $30 \mu \mathrm{m}$ in tip was put concentrically into the cylinder with $80 \mathrm{~mm}$ diameter.

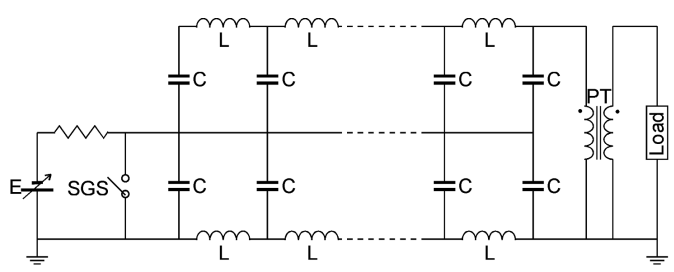

Fig. 1. The schematic diagram of the pulsed power generator.

\subsection{Discharge treatment and gene analysis}

After pouring sterilized distilled water (SDW) into the discharge chamber, red alga (50 mg) was put into the discharge chamber with tweezers. After firing of $\mathrm{B}-\mathrm{PFN}$, the underwater streamer-like discharge was generated in the discharge chamber. At that time, red alga 


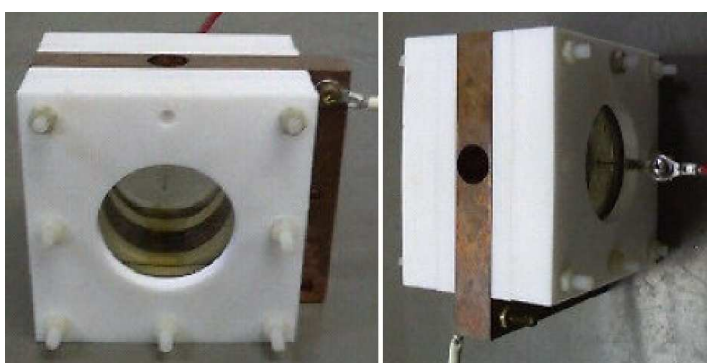

Fig. 2. Discharge chamber.

in the chamber was treated. After applying streamer discharge treatment, total RNA was isolated from leafy gametophytes with Sepasol RNA I Super kit (Nacalai Tesque, Kyoto, Japan) and then total RNA was processed with reversed transcription-polymerase chain reaction (RT-PCR). RT-PCR is a technique for amplifying RNA. At first, the RNA is reverse transcribed into complimentary DNA (cDNA), and then the cDNA is amplified using polymerase chain reaction (PCR). The treated cDNA was analysed using electrophoresis. These results showed transcriptional activation level of retrotransposons and functional genes. For digitizing the electrophoresis results, they were quantified using software TRNSFRM3. The elongation factor gene $(P y E F)$ which has stable transcription even under stress conditions was used as reference level of activation.

\section{Results and discussion}

In the work, four retrotransposons of two copia-like retrotransposons (PyRE1, PyRE2), and two SINE-like elements (PySN1, PySN2), and six functional genes, bromoproxidase $(P y B P)$, superoxide dismutase $(P y S O D)$, and protease gene family $(C l p B 1, C l p P 1, C l p B 2, C l p P 2)$ were isolated from the red alga and mRNAs accumulation levels at $4 \mathrm{~h}$ after applying discharge treatment were investigated by RT-PCR. Figure 3 shows the results of electrophoresis for four retrotransposons and the graph
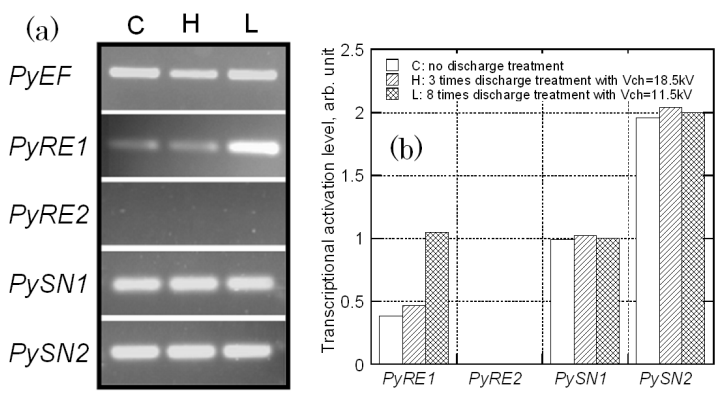

Fig. 3. The effect of pulse streamer discharge on retrotransposon. (a) Electrophoresis photos. (b) Digitized results.

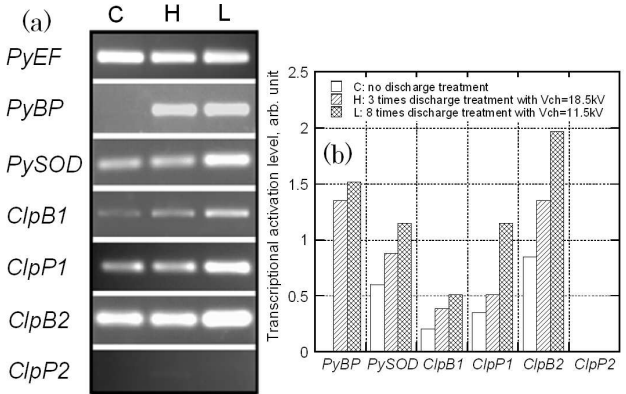

Fig. 4. As in Fig. 3 for functional genes.

digitized of electrophoresis result. In Fig. 3, C (control), $\mathrm{H}$ (high) and $\mathrm{L}$ (low) mean no discharge treatment, 3 times discharge treatments with higher charging voltage $(18.5 \mathrm{kV})$ to B-PFN and 8 times treatments with lower charging voltage $(11.5 \mathrm{kV})$. From Fig. 3, electrophoresis results and the digitizing data showed transcriptional levels of a copia-like retrotransposon, PyRE1 was activated by the streamer-like discharge treatment. In the case of 8 times discharge treatments, L, PyRE1 transcriptional level was activated efficiently. On the other hand, transcriptional levels of PyRE2, PySN1, and PySN2 elements were not activated. Figure 4 shows the results of electrophoresis for six functional genes and the graph digitized of electrophoresis result. The transcriptional levels of five functional genes, PyBP, PySOD, ClpB1, $C l p P 1, C l p B 2$ were activated by the streamer-like discharge treatment. As same in PyRE1, in the case of 8 times discharge treatments, L, PyBP, PySOD, ClpB1, $C l p P 1$ and $C l p B 2$ transcriptional levels were activated efficiently. On the other hand, no activation of transcriptional level of $C l p P 2$ element was shown in Fig. 4.

\section{Conclusions}

A copia-like retrotransposon gene, PyRE1 and functional genes, $P y B P, P y S O D, C l p B 1, C l p P 1$ and $C l p B 2$ were activated by applying pulsed streamer-like discharge in water. The results suggest that the pulsed streamer-like discharge can be used as a useful tool to activate retrotransposon genes and to enhance expression of functional genes.

\section{References}

[1] K. Kuwano, Y. Aruga, N. Saga, Plant Sci. 116, 117 (1996).

[2] M.A. Grandbastien, H. Lucas, C. Mhiri, J.B. Morel, S. Veruhettes, J.M. Casacuberta, Genetica 100, 241 (1997).

[3] N. Pourtau, B. Lauga, C. Audéon, M.A. Grandbastien, P. Goulas, J.C. Salvado, Plant Sci. 165, 983 (2003).

[4] S. Takeda, K. Sugimoto, H. Otsuki, H. Hirochika, Plant Mol. Biol. 36, 365 (1998).

[5] B. Sun, M. Sato, J.S. Clements, J. Electrostat. 43, 115 (1998). 